\title{
Imported Schistosomiasis
}

\author{
J. Uberos and A. Jerez-Calero \\ Pediatrics Service, Universitary Hospital "San Cecilio", Granada \\ Spain
}

\section{Introduction}

Schistosomiasis is a parasitic disease caused by a trematode helminth of the genus Schistosoma. Of the latter, only six species affect humans: S. haematobium, S. mansoni S. japonicum S. intercalatum, S. mekongi and S. malayensis. It is also termed bilharziasis, after its discoverer, the German doctor Theodor Maximilian Bilharz. In 1851, during his work at the Egyptian Department of Hygiene, he discovered this new class of helminth in the vena cava blood of patients who had died of haematuria.

\section{Biological cycle of the genus schistosoma}

Schistosoma has a cylindrical body, ending in two oral suckers. The adult forms are hematophagous, and are found in the venous plexuses, especially the mesenteric or perivesical, in the definitive host, where the male-female pairing takes place. The female migrates to the capillaries and venules, where 100-1000 eggs a day may be deposited. These eggs, in turn, migrate through the walls of the vessels in which they were deposited, reaching the tissues and then either the intestinal lumen or the urinary bladder lumen. Finally, they emerge in the faeces or the urine, respectively. There are two main forms of schistosomiasis: intestinal and urogenital. Among the species that parasitise humans, only $S$. haematobium affects the urogenital apparatus, with the other species normally producing intestinal parasitisation.

Transported in the faeces or urine, the embryo eggs contaminate stagnant water in rivers, lakes and irrigated fields, where in a few hours they hatch and release the miracidia. The latter penetrate and parasitise certain snails that inhabit the fresh water and constitute the intermediary host. Each species of Schistosoma parasitises a particular genus of snail, as follows:

- S. Haematobium: Bulinus, Physopis, Planorbis.

- S. mansoni: Biomphalaria.

- S. japonicum: Oncomelania.

- S. mekongi: Tricula aperta.

- S. intercalatu: Bulinus.

- S. malayensis: Tricula aperta.

Within the snail, they mature for approximately 6 weeks to become cercariae (with a bifurcated tail and penetration glands), which are then released back into the water. Humans can become infected by walking, bathing or washing in water contaminated by 
cercariae, which adhere to the skin and penetrate it. In this process, they lose their tails and are transformed into schistosomulae, which reach the bloodstream and are systemically distributed, reaching the lungs and finally the liver, where they mature into the adult form and attach themselves in their definitive location, the mesenteric or vesical venous plexuses, and thus the cycle is completed. The period between the cercariae penetrating the skin and the beginning of egg production from adult worms is 4-7 weeks. Adult worms can live in the venous plexuses for 20-30 years. Direct transmission between persons does not take place, as the participation of the snail in the biological cycle of this trematode is absolutely necessary.

\section{Geographic distribution}

Schistosomiasis is the second most prevalent tropical disease, after malaria, and has been included in the World Health Organisation list of 'forgotten' tropical diseases. Its geographic distribution varies according to the species of Schistosoma, but it is endemic in 74 countries. Recent studies have calculated that over $95 \%$ of cases are concentrated in Africa, especially in sub-Saharan Africa (80\%), which is explained by the particular socio-ecological conditions prevailing in these areas, with widespread poverty and a virtual absence of populational health systems. Other endemic areas include Central and South America, the Middle East, and SE Asia (Gryseels et al., 2006).

Worldwide, 800 million people are at risk and over 200 million have been infected, most of them in poor communities without access to clean drinking water or adequate sanitation. Of these, 20 million suffer severe consequences of the infection, although, on the other hand, 120 million are asymptomatic. It has been estimated that intestinal schistosomiasis causes, in Africa alone, over 200,000 deaths a year (130,000 due to portal hypertension).

These are agricultural populations, where contact with infected fresh water occurs constantly, when people walk in stagnant water, or swim in rivers or lakes. The construction of new dams and the extension of irrigation systems are known to multiply the risk of contracting this disease (Steinmann et al., 2006). Children, who may play in infected water, and who often suffer a hygiene deficit, are particularly vulnerable. Also at risk are women who carry out domestic tasks such as washing clothes in these waters. It has been estimated that in chronic cases of vaginal schistosomiasis, the risk of HIV infection is multiplied threefold (Ndhlovu et al., 2007).

The WHO strategies for controlling schistosomiasis are centred on reducing cases of the disease via periodic, focused treatment with praziquantel. This is a cheap and effective drug, costing only $€ 0.08$ per dose. Nevertheless, it may still be expensive for the health administrations of some developing countries. Outbreaks of schistosomiasis can be identified by haematuria screening of schoolchildren and, in cases of epidemic, treating the entire community at risk. Annual doses of praziquantel are sometimes recommended in these areas at high risk of re-infection, and contribute to reducing the severity of symptoms among the chronically sick, and minimising the consequent multi-organ dysfunction. In Egypt, China and Uganda, in just a few years, this approach has reduced the prevalence and intensity of parasitisation, and anaemia (Global Network for Neglected Tropical Diseases; Hotez et al 2010). 
Therefore, the international community must be made aware of the social and economic magnitude of the problem. International donations are urgently needed to control this disease, which by its prevalence and severity is greatly hampering the economic advance and sustainable development of many African countries (Baker et al., 2010). Nevertheless, praziquantel is not a panacea, and actions in endemic countries should also include integrated strategies aimed at the identification, monitoring and surveillance of these tropical diseases which, because they are so infrequent in developed countries, are frequently overlooked or ignored (Utzinger et al., 2010). This is the background to the creation of the Global Network for Neglected Tropical Diseases, an initiative aimed at supporting developing countries in their fight against 'forgotten' diseases that affect 1400 million people all over the world. This organisation was inaugurated in 2006 and has its headquarters at the Sabin Vaccine Institute in Washington.

In Africa, refugee movements and migration toward cities, as well as to countries in the developed world, are introducing this disease into previously unaffected areas. Increasing population numbers and the corresponding greater demand for energy and water often give rise to development plans and environmental actions that also contribute to increasing the transmission of the disease. The boom in ecotourism and journeys outside controlled areas are also provoking an increase in the number of tourists with imported schistosomiasis, among all the neglected tropical diseases (López-Vélez et al, 2008).

The GeoSentinel Surveillance Network carried out a transversal study of affected travellers who had visited endemic schistosomiasis areas between 1997 and 2008. Confirmed cases were communicated in 12 countries belonging to this worldwide surveillance network. In a total sample of 25,240 , some 410 persons (16 per thousand) were found to be suffering from schistosomiasis; 37\% were European, $24 \%$ North American, 24\% Asian and 15\% from Oceania. Over 80\% of those affected had acquired this disease in Africa, mainly in sub-Saharan Africa. There were some differences depending of demographic characteristics and the type of travel. Male travelers and travelers $<45$ years of age were more likely to be diagnosed with schistosomiasis relative to another illness. When the data were controlled for age and sex, those traveling for missionary or other volunteer work were twice as likely to be diagnosed with schistosomiasis as were tourist and other types of travelers. Business travelers were less likely to be diagnosed with schistosomiasis than were tourists and other types of travelers. Individuals traveling as expatriates, regardless of the type of travel, were twice as likely to be diagnosed with schistosomiasis as those staying for shorter times and staying in hotels. Having a pre-travel consultation was also associated with being diagnosed with schistosomiasis. Sixty-three percent of the 401 ill returned travelers with schistosomiasis presented to a GeoSentinel clinic within six months after travel with a median time-to-presentation of six weeks (IQR 2-12 weeks; The median duration of travel for this group was 13 weeks (IQR 4-33 weeks). One hundred ten (27\%) travelers presented to a GeoSentinel clinic more than 6 months after travel. Time-to-presentation was between 6 months and two years for $73(18 \%)$, three to four years for $16(4 \%)$, and five or more years for $18(4 \%) .41 \%$ of the 319 travelers who were asymptomatic at presentation, were diagnosed with schistosomiasis. The most commonly reported symptoms Gastrointestinal symptoms were fever, genitourinary symptoms, and fatigue. Returned travelers with schistosomiasis were more likely to present with fever than those who were ruled-out for 
schistosomiasis. Those with S. haematobium and S. mansoni infections presented with more respiratory symptoms than did those ruled-out for schistosomiasis. Moreover, returned travelers with schistosomiasis who were seen within six months of travel more often presented with fever and respiratory symptoms compared with those who presented later (Nicolls et al., 2008).

Imported travel-related infections were studied in a retrospective and descriptive study of travelers returning from the tropics and attended at the Tropical Medicine Unit, Infectious Diseases Department of Hospital Ramon y Cajal in Madrid (Spain) during the period January 1989 to November 2006. The total number of ill travelers analyzed was 2,982; 1387 traveled to Sub-Saharian Africa and 44 of them were diagnosed with schistosomiasis (3\%). A total of $98 \%$ of patients with schistosomiasis had traveled to Sub-Saharian Africa and only $2 \%$ to other geographical areas. (Zamarrón et al, 2010)

Many centres in Australia routinely screen African refugees for schistosomiasis using serology, and in some cases with faeces and urine examination for schistosome ova. There were a seroprevalence of $38 \%$ for 653 African refugee adults and children arriving in Australia, predominantly from Liberia, Burundi, Tanzania and Sudan. A multivariate analysis of the Newcastle data showed that schistosomiasis serology was much more likely to be positive in those from East Africa (OR 14.5) and West Africa (OR 5.5) than from the Sudan region (OR 1.0). (Davis et al., 2007) In those who had faeces and urine examination in these cohorts, urinary schistosomiasis is uncommon, with $>95 \%$ of those with positive microscopy having S. mansoni in faeces (Australasian Society for Infectious Diseases, 2009).

\section{Clinical signs}

The clinical manifestations of the disease are produced by the body's inflammatory reaction to the eggs and larvae, as the adult worm eludes the humeral and cell-mediated immune response. In the blood vessels, attempts at migration through the walls may produce haemorrhages and inflammatory processes. When larvae become lodged in the intestinal or vesical tissues, they may provoke granulomas, which in time become calcified. In the intestinal forms, some of the eggs are transported to the portal venous plexuses and may become trapped in the liver, producing periportal fibrosis.

\subsection{Acute schistosomiasis}

In the cercariae entry zone, pruriginous skin lesions of a macular or macular-papular type may appear, the so-called "swimmer's itch". In persons who have previously been exposed and therefore are sensitised, itching may persist and wheals may be suffered for some days. In endemic zones, this cercarial dermatitis may remain unnoticed.

Katayama fever is a systemic hypersensitivity reaction that appears at 2 to 6 weeks after exposure to and penetration by the cercariae. It is relatively infrequent among the endemic population (due to infradiagnosis or intrauterine sensitisation) and more common among persons who have not been previously exposed, such as tourists who come into contact with contaminated water. It is provoked mainly by the species $S$. mansoni and S. japonicum, and less frequently so by $S$. haematobium. The most obvious symptoms coincide with the eggdepositing phase and consist of fever, shivering, swollen lymph nodes, headaches, abdominal pain, bloody diarrhoea and painful swelling of the liver. In addition, respiratory 
symptoms such as coughing, chest pain or breathing difficulties are often encountered. This process is normally self-limiting in time, with a progressive improvement after several weeks. Only complications derived from massive infestation may lead to death (approximately $0.5 \%$ of symptomatic cases).

\subsection{Chronic schistosomiasis}

Lesions arising from chronic infection are due to eggs that remain trapped in the tissues over a long period of time. These eggs release proteolytic enzymes that typically provoke eosinophilic and granulomatous inflammatory reactions, which are progressively followed by fibrotic deposits. This commonly affects individuals who are continually exposed to infection in poor rural areas. Apart from digestive or urinary manifestations, other less common locations have been reported, such as the lungs, the genitals or the brain.

Among children, the clinical presentation of chronic schistosomiasis varies widely, although cases with a high degree of parasitisation normally present anaemia, malnutrition and delayed growth. Many of these paediatric cases are reversible with appropriate treatment. Moreover, the vertical transmission of the disease by pregnant women has been reported.

\subsection{Urogenital schistosomiasis}

This condition is produced solely by the species S. haematobium. Haematuria is a classical, initial sign of urogenital schistosomiasis. In advanced cases, a frequent sign is urinarybladder fibrosis, which may in turn provoke hydronephrosis. Symptoms and signs include dysuria, urgent urination, terminal microscopic and macroscopic haematuria, secondary urinary infections and non-specific pelvic pain. Approximately half of all infected children develop moderate-severe pathology of the urinary tract, including pyelonephritis, urethral hydronephrosis, vesical pseudo-papillomas, nephrotic syndrome, etc. Women with urogenital schistosomiasis may present genital lesions, vaginal haemorrhages, dyspareunia or vulvar nodules. Vaginal alterations provoked by chronic schistosome infection may triple the risk of HIV infection. Among men, it may cause disorders of the seminal vesicles and of the prostate and consequent male infertility. Chronic renal insufficiency and squamous cell carcinoma of the bladder are other possible long-term complications.

\subsection{Intestinal schistosomiasis}

This is produced by the species S. mansoni, S. japonicum, S. intercalatum and S. mekongi. The areas where it is most commonly found are the mucous membranes of the large intestine and the rectum, where it produces a granulomatous inflammation, with pseudopolyps and microulcerations. Clinical manifestations include colicky abdominal pain, diarrhoea and, sometimes, gastrointestinal blood loss.

\subsection{Hepatic schistosomiasis}

Hepatic alterations may range from mild inflammation to severe fibrosis. The initial inflammation appears as a periportal pre-sinusoidal reaction to the presence of the eggs and is an important cause of hepatomegaly among children and adolescents (it appears in up to 
$80 \%$ of infected children). In the initial stages, most cases remain asymptomatic. Chronic hepatic schistosomiasis or fibrosis develops years after the original infection and is found more in young adults with intensive, long-term infections and perhaps a certain degree of genetic predisposition. It is the result of a massive deposit of collagen in the periportal spaces, which produces pipe-shaped pathognomonic periportal fibrosis, as described by Symmer (5-10\% of patients). In advanced phases, hepatomegaly is frequent, as are ascites and portal hypertension, which with their secondary complications may lead to death (King and Dangerfield-Cha, 2008). Associated with these cases, in addition, may be splenomegaly, esophageal varices and severe haematemesis.

Sometimes other organs are compromised by the systematic embolisation of eggs or following anomalous migration of the adult schistosome. In the lungs, it is not uncommon for this to cause coughing, breathing difficulties and, finally, pulmonary hypertension or cor pulmonale. In the nervous system, the medulla may be the site of infection by $S$. mansoni or S. haematobium, provoking transverse myelitis or affecting the medullary cone and the cauda equina. Encephalic effects are only produced by S. japonicum and usually involve epileptic symptoms or diffuse encephalitis, following the formation of cerebral granulomas.

\section{Diagnosis}

In Spain and in other countries bordering the Mediterranean, immigration has increased in recent years. According to data published by the Spanish National Institute of Statistics, in the year 2000 the immigrant population constituted $2.7 \%$ of the population, while in 2007, there were 4.5 million immigrants, making up $12.7 \%$ of the population. Over $50 \%$ of the immigrant population comes from areas with a high prevalence of schistosomiasis: $39 \%$ from Latin America and 14\% from sub-Saharan Africa (Instituto Nacional de Estadística, 2010). In Spain and other European countries, the absence of intermediate hosts, together with generally good healthcare conditions, makes infection here almost impossible, and there is no risk of transmission within the population. The high prevalence of schistosomiasis in some countries in sub-Saharan Africa, together with rising rates of immigration, has led to more and more cases of urinary schistosomiasis being reported in many European countries. Investigation of cases of long-term haematuria often reveals a background of journeys to endemic areas, and in such cases a fresh urine examination may be requested. These data demonstrate the need to consider schistosomiasis in the differential diagnosis of hematuria in daily practice in primary care in developed countries. There are references to urinary schistosomiasis among immigrants arriving from endemic areas or following prolonged stays in the above countries, involving contact with contaminated water (Uberos et al., 2010).

During the stage of larval invasion or migration, clinical symptoms respond to the laying of eggs and the formation of soluble antigens. This is characteristic of S. mansoni and may last up to three months. It is characterised by periportal fibrosis, portal hypertension and enlargement of the liver and spleen, with high fever, fatigue, urticaria, swollen lymph nodes and eosinophilia. There may also be abdominal pain, weight loss and diarrhoea. The presence of eosinophilia among the immigrant population is almost always due to infection by helminths (Corachan, 2002). Eosinophilia is defined as a total eosinophil count exceeding 
$440 \times 10^{6} / \mathrm{ml}$. Various studies (Bierman et al., 2005) have estimated that eosinophilia affects $40-65 \%$ of patients. When the egg-laying stage is attained, the clinical pattern is characterised by fever, coughing, shivering, urticaria, abdominal pain, adenopathy and enlarged spleen, which occurs at 1 to 2 months following infection and may last for over 3 months. This symptomatic complex is known as the Katayama syndrome.

Months or years after the beginning of parasitisation there may occur granulomatous reactions and fibrosis in the organs affected, including mild effects in the gastrointestinal tract with epigastralgia and diarrhoea, or chronic polyps, bloody diarrhoea, haematemesis and enlarged spleen. In the urogenital tract, S. haematobium is associated with haematuria, pollakiuria, suprapubic pain and thickening of the vesical wall, hydroureter or hydronephrosis. Cases of neuroschistosomiasis have also been reported. Histological lesions of the schistosomes result from the deposition of eggs in the tissues, where they may provoke the formation of granulomas, hyperplasia of the mucous membranes and the formation of nodules and polyps that tend to ulcerate and bleed.

In many cases, there is haematuria, with the emission of several drops of fresh blood at the end of every urination. In general, no fever or dysuria is reported. In Spain, the existence of parasitisation by $S$. haematobium is frequently revealed when patients seek treatment for persistent haematuria, which is addressed by serial urine culture and empirical antibiotic treatment. In these cases, careful consideration of the patient's background, together with evidence of journeys made in recent months to endemic zones, should guide the physician's diagnosis.

Classic parasitological techniques (analysis of sediment) are available to any laboratory, are easy and cheap, but time consuming and require experience of the observer. They also have a low sensitivity for diagnosis. Direct parasitological diagnosis is performed by examination of eggs in the faeces (the Kato-Katz technique) or in 24-hour urine. The presence of eggs in the intestinal mucosa may also be investigated, by biopsy. At present, the Kato-Katz technique is the most frequently recommended, and this is preferred by the WHO both for individual diagnostic studies and for epidemiological investigation. Moreover, it is used to determine the prevalence and intensity of soil-transmitted helminthiasis. In addition, it is useful for establishing the relation between the number of eggs in the faeces and the quantity of adult parasites in the infected host. This information is essential for determining the parasite load in the patient.

Schistosomiasis may be hard to diagnose in patients with a low parasite load and scant excretion of eggs, when microscope identification is difficult. Live parasites produce and excrete many protein compounds in the different phases of the parasitic cycle. Immunodiagnostic techniques are based on the detection of these proteins or antibodies formed against them, being more sensitive than conventional ones. The detection of circulating antigens difference between active and past infection, and correlate with the intensity of infection, morbidity and severity.

Serological diagnosis may be useful for cases of acute schistosomiasis in areas where it is not endemic (Tarp et al., 2000). When serological results are positive, another search for Schistoma eggs should be made. When the results of the serological examination are negative, it can be assumed that in non-endemic areas the risk of schistosomiasis is very low. The determination of antibodies allows early diagnosis of the disease. While not opposed to an actual infection of another pass, since the antibodies can be kept at high titers for months or even years. Serology requires a period of six weeks for positivisation. 
Serological diagnosis can be carried out using indirect immunofluorescence techniques and ELISA. The following in vivo precipitation techniques are applied:

a. Vogel-Minning technique (circum-cercarial precipitin test-CCPT). With this technique, the cercariae are brought into contact with the patient's serum. If the serum produces specific antibodies, the presence of a highly specific precipitate can be observed, due to the intervention of cercarial metabolic antigens.

b. Oliver-Gonzalez technique (circum-oval precipitin test - COPT). This technique is identical to the previous one, except that live eggs are used.

By means of these techniques, it is possible to determine the evolution of the disease. For example, for recent schistosomiasis, the CCPT technique is used.

Image testing (ultrasound imaging, urography, etc.) can be very useful. For digestive schistosomiasis, rectal or liver biopsy and colonoscopy may also be useful. Most methods used to evaluate the success of interventions, such as egg counting, measure the level of infection but do not provide direct evidence of the pathological changes induced by the infection. Ultrasound is an excellent tool for assessing the secondary pathological alterations produced by the infection. The prevalence and severity of the pathological lesions detected by ultrasound are related to the intensity of the infection, evaluated by the quantity of eggs eliminated in the faeces or urine. In endemic zones, the maximum rate of morbidity is observed among children aged 7-14 years, while in non-endemic zones, the disease is diagnosed in all series following an evolution of 2-3 months. The lesions most often diagnosed by ultrasound include hydronephrosis, the presence of intravesical masses, thickening of the bladder wall and calcification, with the appearance of hydronephrosis being associated with the poorest prognosis. Some series have identified lesions to the female genital apparatus, with masses identifiable by ultrasound examination. An association has been shown between epidermoid carcinoma of the bladder and $S$. haematobium (Corachan, 2002).

Ultrasound may be useful for diagnosing periportal fibrosis and portal hypertension (dilation of the portal and splenic veins and the portosystemic collaterals). Treatment for intestinal schistosomiasis generally produces an improvement in the signs of infection, including reduced bloody diarrhoea, intestinal polyposis, hepatosplenomegaly and periportal fibrosis.

\section{Treatment}

Following confirmation of urinary schistosomiasis, the treatment normally prescribed is oral praziquantel at a dose of $600 \mathrm{mg} / 12 \mathrm{~h}$, in two doses. The analytical analysis is repeated, 15 days following this treatment, by determining the eosinophil count in blood and by a serial examination of fresh urine samples, on three consecutive days, with the presence of $S$. haematobium in urine being discounted when haemogram results are normal. S. haematobium is the most frequent cause of haematuria in countries where this disease is endemic. All protocols for treating immigrant children include the search for S. haematobium when persistent haematuria is reported. We believe this search should be expanded to cases of persistent haematuria when there is a case history of bathing or swimming in rivers in zones endemic for S. haematobium. The existence of haematuria is related with the presence of viable eggs in the bladder wall, which in long-term processes is in turn related with the presence of granulomas in the vesical submucosa and the distal part of the urethra (Tzanetou et al., 2007). In cases of repeated, severe infection, the urethral mucosa are also 
affected, and this may evolve to obstructive uropathology and hydronephrosis. The preferred treatment in this case is praziquantel at a dose of $40 \mathrm{mg} / \mathrm{kg} /$ day, in two doses at intervals of 12 hours. At this dose, the cure rate at six weeks is $88 \%$. The administration of a second dose of praziquantel at 4-6 weeks increases the cure rate to 100\% (Midzi et al., 2008). To prevent resistance, some authors recommend a dose of praziquantel at $60 \mathrm{mg} / \mathrm{kg} / 8 \mathrm{~h}$, for three days (Botros et al., 2005). During their immature stages, the schistosomes are moderately resistant to praziquantel, becoming more sensitive on maturity, when the eggs are laid. This means that during the first 10-12 weeks following infection by S. haematobium, praziquantel may not be effective (Botros et al., 2005). As an alternative treatment for $S$. haematobium, metrifonate or niridazol may be applied.

Metrifonate is a reversible competitive inhibitor of the acetylcholinesterase enzyme, including the human enzyme. As such, it is a parasympathetic mimetic agent that prolongs the action of the parasympathetic nervous system, and in which the neurotransmitter is acetylcholine. It performs selective, variable schistosomocidal activity against $S$. haematobium, provoking its partial metabolisation into dichlorvos, an active anti-cholinesterase compound. The cholinesterase of the schistosome is more susceptible to this metabolite than that of the human host, but at the recommended treatment dose, transitory reductions in cholinesterase activity, both in plasma and in erythrocytes can be observed. Despite early concerns expressed about its possible toxicity, metrifonate is well tolerated and has been widely employed, with good results in important treatment programmes. A dose of $7.5 \mathrm{mg} / \mathrm{kg}$, taken on three occasions, at intervals of two weeks, produces a $40-80 \%$ cure rate. Mass chemotherapy should not be undertaken in communities recently exposed to insecticides or other agricultural chemicals with an anti-cholinesterase action. Treated patients should not be given depolarizing neuromuscular blocking agents such as suxamethonium until at least 48 hours have elapsed from the time of metrifonate administration. If necessary, to alleviate the symptoms of cholinergic activity, atropine sulphate can be used as a specific antidote (for adults, $1 \mathrm{mg}$ every six hours). This measure does not impair the anti-parasitic action.

Niridazol is absorbed slowly from the gastrointestinal tract in 10-15 hours. It metabolises rapidly during its first pass through the liver, and its metabolites are eliminated in equal quantities in the urine and faeces. Schistomocidal activities are produced because niridazol is not metabolised in the blood. The normal dose for adults infected with $S$. haematobium or S. mansoni is $25 \mathrm{mg} / \mathrm{kg} /$ day, for seven days. For children infected with $S$. haematobium, the treatment duration may be reduced to 5 days if a daily dose of $30-35 \mathrm{mg} / \mathrm{kg}$ is administered. Children infected with $S$. mansoni should be treated with $25 \mathrm{mg} / \mathrm{kg} /$ day for seven days. In bilharziasis caused by $S$. japonicum, the daily dose for both adults and children is 20-25 $\mathrm{mg} / \mathrm{kg}$ for five days.

As the recommended treatment for intestinal schistosomiasis (S. mansoni), both in the acute phase and when the liver and spleen are affected, we propose oxamniquine. This is a tetrahydroquinoline derivative with selective, variable schistosomocidal activity against S. mansoni. Male schistosomes are more susceptible than females, but the females that survive cease to lay eggs after exposure to oxamniquine and become irrelevant from a pathological standpoint. Some varieties are resistant, especially in South America, although these respond to subsequent treatment with praziquantel. Oxamniquine is well absorbed when administered orally and generally metabolises to 
inactive acid metabolites that are eliminated in the urine. It is well tolerated and has been widely used with good results in control programmes carried out in South America.

\section{Control measures against schistosomiasis}

The aim of control programmes is to reduce morbidity. In 1922, after the discovery of the role played by snails in transmission of the disease, strategies were developed consisting in the primary control of snails in highly endemic areas. From 1960, new technologies were discovered to achieve the same goal; on the one hand, niclosamide, was applied to eliminate the snails, and on the other, praziquantel was found to be a safe treatment option with few secondary effects. Countries such as Egypt, with high rates of endemic schistosomiasis, have developed control programmes including annual checkups for school-age children and treatment for all suspected cases, together with programmes to control snail infestation by the drainage and cleaning of all rivers and canals (Salem et al., 2011). Thanks to these measures, by the end of 2002, the prevalence of S. mansoni had been reduced to $2.7 \%$, from the value of $14.8 \%$ recorded in 1993 . Similarly, the prevalence of S. haematobium fell from $6.6 \%$ in 1993 to $1.2 \%$ in 2006 . This method reduced morbidity, but in other areas, improved water supply has also led to low levels of prevalence.

In countries of origin, it seems reasonable that the primary objective in the control of schistosomiasis consists in reducing morbidity, or at least severe forms of the disease. The imported forms of the disease appears early diagnosis key to disease, in environments where the low prevalence of the disease complicates the diagnosis. The importance of history, focusing on the existence of previous residence or travel in endemic areas seems essential.

In the communities of African immigrants, is often found that more than one family member is parasitized and hematuria, if not too intensive, is an inconspicuous sign for the patient or their relatives. This fact illustrates the need to subject these patients to a series of systematic reviews of health in destination countries to establish not only the diagnosis of imported diseases as the present one, but also those others present in our midst and very frequent in the geographic area of origin.

\section{References}

Baker, M.C., E.Mathieu, F.M.Fleming, M.Deming, J.D.King, A.Garba, J.B.Koroma, M.Bockarie, A.Kabore, D.P.Sankara, and D.H.Molyneux. 2010. Mapping, monitoring, and surveillance of neglected tropical diseases: towards a policy framework. Lancet 375:231-238.

Bierman, W.F., J.C.Wetsteyn, and G.T.van. 2005. Presentation and diagnosis of imported schistosomiasis: relevance of eosinophilia, microscopy for ova, and serology. J. Travel. Med. 12:9-13.

Botros, S., L.Pica-Mattoccia, S.William, N.El-Lakkani, and D.Cioli. 2005. Effect of praziquantel on the immature stages of Schistosoma haematobium. International Journal for Parasitology 35:1453-1457.

Corachan, M. 2002. Schistosomiasis and international travel. Clin. Infect. Dis. 35:446-450. 
Gryseels, B., K.Polman, J.Clerinx, and L.Kestens. 2006. Human schistosomiasis. Lancet 368:1106-1118.

Global Network for Neglected Tropical Diseases. http://www.globalnetwork.org/ Acces June, 162011.

Hotez P, Engels D, Fenwick A, Savioli L. 2010.Africa is desperate for praziquantel. Lancet. 376, Issue 9740: 496-8

Instituto Nacional de Estadística. Encuesta nacional de inmigrantes 2007. www.ine.es/inebmenu/mnu_migrac.htm . Acces June, 162010.

López-Vélez R, Pérez Molina JA, Zamarrón P, Pérez de Ayala A. Enfermedades infecciosas importadas por viajeros internacionales a los trópicos. 2008. Ministerio de Sanidad y Consumo. Gobierno de España.

King, C.H., and M.Dangerfield-Cha. 2008. The unacknowledged impact of chronic schistosomiasis. Chronic Illn. 4:65-79.

Midzi, N., D.Sangweme, S.Zinyowera, M.P.Mapingure, K.C.Brouwer, N.Kumar, F.Mutapi, G.Woelk, and T.Mduluza. 2008. Efficacy and side effects of praziquantel treatment against Schistosoma haematobium infection among primary school children in Zimbabwe. Transactions of the Royal Society of Tropical Medicine and Hygiene 102: 759-766.

Ndhlovu, P.D., T.Mduluza, E.F.Kjetland, N.Midzi, L.Nyanga, S.G.Gundersen, H.Friis, and E.Gomo. 2007. Prevalence of urinary schistosomiasis and HIV in females living in a rural community of Zimbabwe: does age matter? Trans. R. Soc. Trop. Med Hyg. 101:433-438.

Nicolls, D.J., L.H.Weld, E.Schwartz, C.Reed, S.F.von, D.O.Freedman, and P.E.Kozarsky. 2008. Characteristics of schistosomiasis in travelers reported to the GeoSentinel Surveillance Network 1997-2008. Am. J. Trop. Med Hyg. 79:729-734.

Zamarrón Fuertes P, Pérez-Ayala A, Pérez Molina JA, Norman FF, Monge-Maíllo B, Navarro M, López-Vélez R. Clinical and epidemiological characteristics of imported infectious diseases in Spanish travelers. J Travel Med. 2010 Sep;17(5): 303-9.

Davis JS, Webber MT. A prospective audit of 215 newly arrived African refugees. J Paediatr Child Health 2007;42:A11

Australasian Society for Infectious Diseases. Diagnosis, management and prevention of infections in recently arrived refugees. Dreamweaver Publishing Pty Ltd. 2009. ISBN 978-0-9750-483-4-4.

Salem, S., R.E.Mitchell, A.El-Alim El-Dorey, J.A.Smith, and D.A.Barocas. 2011. Successful control of schistosomiasis and the changing epidemiology of bladder cancer in Egypt. BJU. Int. 107:206-211.

Steinmann, P., J.Keiser, R.Bos, M.Tanner, and J.Utzinger. 2006. Schistosomiasis and water resources development: systematic review, meta-analysis, and estimates of people at risk. Lancet Infect. Dis. 6:411-425.

Tarp, B., F.T.Black, and E.Petersen. 2000. The immunofluorescence antibody test (IFAT) for the diagnosis of schistosomiasis used in a non-endemic area. Trop. Med. Int. Health 5:185-191.

Tzanetou, K., G.Adamis, E.Andipa, C.Zorzos, K.Ntoumas, K.Armenis, G.Kontogeorgos, E.Malamou-Lada, and P.Gargalianos. 2007. Urinary tract Schistosoma haematobium infection: a case report. J Travel. Med 14:334-337. 
Uberos, J., M.Gamarra, E.Prados, and E.Narbona-Lopez. 2010. Importance of anamnesis in the diagnosis of urinary schistosomiasis.. An. Pediatr. (Barc.) 73:214-216.

Utzinger, J., E.K.N'goran, C.R.Caffrey, and J.Keiser. 2010. From innovation to application: Social-ecological context, diagnostics, drugs and integrated control of schistosomiasis. Acta Trop. (doi:10.1016/j.actatropica.2010.08.020). 


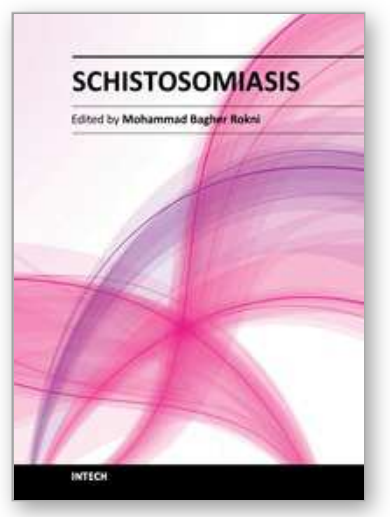

\author{
Schistosomiasis \\ Edited by Prof. Mohammad Bagher Rokni
}

ISBN 978-953-307-852-6

Hard cover, 310 pages

Publisher InTech

Published online 13, January, 2012

Published in print edition January, 2012

In the wake of the invitation by InTech, this book was written by a number of prominent researchers in the field. It is set to present a compendium of all necessary and up-to-date data to all who are interested.

Schistosomiasis or blood fluke disease, also known as Bilharziasis, is a parasitic disease caused by helminths from a genus of trematodes entitled Schistosoma. It is a snail-borne trematode infection. The disease is among the Neglected Tropical Diseases, catalogued by the Global Plan to combat Neglected Tropical Diseases, 2008-2015 and is considered by the World Health Organization (WHO) to be the second most socioeconomically devastating parasitic disease, next to malaria. WHO demonstrates that schistosomiasis affects at least 200 million people worldwide, more than 700 million people live in endemic areas, and more than 200.000 deaths are reported annually. It leads to the loss of about 4.5 million disability-adjusted life years (DALYs).

\title{
How to reference
}

In order to correctly reference this scholarly work, feel free to copy and paste the following:

J. Uberos and A. Jerez-Calero (2012). Imported Schistosomiasis, Schistosomiasis, Prof. Mohammad Bagher Rokni (Ed.), ISBN: 978-953-307-852-6, InTech, Available from:

http://www.intechopen.com/books/schistosomiasis/imported-schistosomiasis

\section{INTECH}

open science | open minds

\author{
InTech Europe \\ University Campus STeP Ri \\ Slavka Krautzeka 83/A \\ 51000 Rijeka, Croatia \\ Phone: +385 (51) 770447 \\ Fax: +385 (51) 686166 \\ www.intechopen.com
}

\author{
InTech China \\ Unit 405, Office Block, Hotel Equatorial Shanghai \\ No.65, Yan An Road (West), Shanghai, 200040, China \\ 中国上海市延安西路65号上海国际贵都大饭店办公楼405单元 \\ Phone: +86-21-62489820 \\ Fax: $+86-21-62489821$
}


(C) 2012 The Author(s). Licensee IntechOpen. This is an open access article distributed under the terms of the Creative Commons Attribution 3.0 License, which permits unrestricted use, distribution, and reproduction in any medium, provided the original work is properly cited. 